\title{
Gamma Correction and Absolute Coordinate Residual Compensation for Triangular Patterns Phase Shifting Profilometry
}

\author{
Yang Wang, Jianying Fan*, Yao Feng, Huijuan Yuan and Shuang Yu \\ The Higher Educational Key Laboratory for Measuring \& Control Technology and \\ Instrumentations of Heilongjiang Province, Harbin University of Science and \\ Technology, Harbin, China \\ *Corresponding author: ceaser@126.com \\ Abstract
}

\begin{abstract}
Triangular patterns phase shifting profilometry is method of structured light whose three-dimensional reconstructions are susceptible to error from gamma distortion. In practice, the luminance nonlinearity caused by the gamma distortion effect of a digital projector and a digital camera yields undesired fringe intensity changes, which substantially reduce the measurement accuracy, and while the effects of the gamma distortion diminish with an increasing number of employed phase-shifted patterns. A gamma correction method based on the pre-coding of projected grating is proposed to reduce the measurement error caused by the gamma distortion. (By) applying an appropriate pre-coding value to the projected patterns, the gamma distortion effect is attenuated and the accuracy is enhanced. The linear least square is compensated the absolute coordinate residuals still existing after gamma correction. To verif the presented nethod in this paper, a 3D shape measurement experimental system is constructed using digital video projector and CCD camera. The experiment results shown that, the gamma pre-coding correction reduce the maximum residuals error of absolute coondinate by $72.2 \%$. After the linear least square compensation, the maximum absolute coordinale residual is only $6.1 \%$ of without any correction or compensation. The reconstulction surface of a complex curve surface is hardly any waviness, which is clearly noted in the reconstruction of the same tested object without any correction or compensation.
\end{abstract}

Keywords; Phase-shifting Profilometry, Triangular Patterns, Gamma distortion, Gamma Correction, Linear Least Square

\section{Introduction}

Phase Shifting Profilometry (PSP) is one of the leading structured light methods for threedimensional (3D) shape measurement [1], which is acquired the distortion fringe image by projecting structured-light patterns onto the objects, and by modulating the object surface of interest [2]. Compared to other methods, PSP has the advantage of high resolution, high measurement speed; less sensitive to the surface reflectivity variations and the object can be measured point by point [3].

More recently, the Triangular Patterns PSP (TPPSP) [4, 5], based on digital fringeprojection, intensity-ratio, and phase-shifting profilometry, has recently been developed. Instead of calculating the phase distribution as in the traditional phase measuring profilometry methods, this technique retrieves the 3D information of the measured object by computing the intensity ratio from the captured distortion triangular fringe images. The advantages of this 
technique are that it uses a simple intensity-ratio computation to measure the $3 \mathrm{D}$ object surface compared to traditional sinusoidal and trapezoidal phase shifting methods [6].

However, the measurement accuracy of PSP is usually affected by many error sources such as the phase-shift error, camera noise and nonlinearity, vibration, and speckle noise [7]. By used in a PSP, the commercial Digital Video Projector (DVP) and the CCD camera are generally fabricated to be nonlinear devices for a better visual effect [8], and the gamma distortion of DVP and CCD camera pairs is the most important factor for the measurement accuracy of 3D shape measurement. Over the years, many studies have performed on understanding and reducing the phase shifting error of gamma distortion. All these methods could be categorized three types.

The first uses a low-pass filter. This type of method was originally proposed by Carocci et al. for measurement systems based on the digital fringe projection [9]. Defocusing [8] method is belonging to this type. The second method is phase error compensation by interpolation fitting and Look-Up Table (LUT). Chen, et al were proposed method adopts smoothing spline approximation to precisely extract the specific system phase eyror from the reference phase according to the system's phase distribution properties [10]. But the spline interpolation fitting is multiple iterations, and time-consuming is expensive, LUT has been proved that the shape waviness is associated only with the nonlinearity of the gamma curve of the DVP and CCD camera pair [7]. This intensity LUT can be constructed by either measuring the entire projected intensity levels or analyzing the fringe images statistically. Another LUT is presented by Zhang and Yau [11], and without pre-conppyting gamma. The accuracy of phase compensation depended on the length of the LET because the measured phase was a realnumber data set involving interpolation. The third method is gamma curve correction. Guo et al. employed statistical methods of analyze and correct for gamma distortion by framing gamma distortion as an uncertainty problem [12]. In their experiment, high-frequency fourstep PMP was adopted for a gamma value of 1.4802, which was not a particularly challenging problem. Thang et al. were presented a pre-coding process is applied to correction the system for actual PSP system [13]. This nethod had advantages, such as simple and suited to conventional few-step phase-shifting algorithm. But it was using a relatively large phaseshifting step (e.g., 20) to deternine the correct phase distribution.

In this paper, we propose a Triangular Patterns Phase Shifting Profilometry (TPPSP) mathematical gamma nodel for based on [13], but only using six gamma-pre-coding uniform luminance patterns for detecting the gamma value involved in the DVP and CCD camera pair. Further the Absolution Coordinate Distribution Compensation (ACDC) obtained by linear least square is using for reducing the phase shifting error after gamma correction. In our experiment the Maximum Absolute Coordinate Residuals error (MACR) of TPPSP can be reduced by $72.2 \%$ when our gamma correction method is applied with gamma pre-decoding, and after ACDC processing MACR is only $6.1 \%$ of without any correction or compensation.

\section{Principle of Gamma Pre-coding and Phase Distribution Least Square}

\subsection{Principle of gamma pre-coding}

In TPPSP, as shown in Figure 1, a series of ideal triangular fringe patterns, which grayscales distribution of patterns as shown in Figure 2, are generated by a computer and projected to the 3D object surface using a DVP. The CCD camera captures the reflected intensity from 3D object surface for further processing. Owing to the nonlinear gray-scale response of a TPPSP system including the overall influences of the DVP, object surface reflectivity, environment brightness, and the CCD camera, the captured fringe images will deviate from its ideal triangular waveform. 


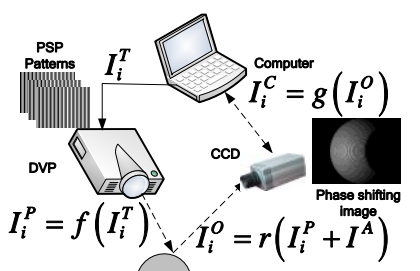

Figure 1. PSP measurement systems

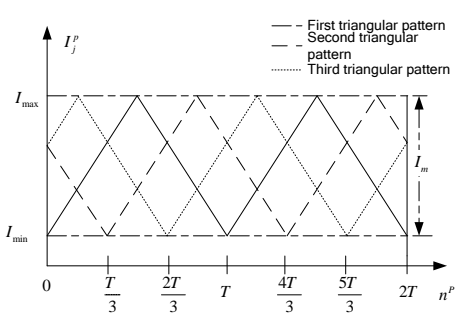

Figure 2. gray-scale distribution of the 3-step TPPSP patterns

Typically, a pixel of the projected TPPSP pattern generated by a computer is expressed as

$$
I_{i}^{T}\left(n^{T}, m^{T}\right)=\left\{\begin{array}{l}
I_{\min }+\frac{2 I_{m}}{T} \bmod \left(n^{T}+\frac{i-1}{3} p, p\right) \\
I_{\max }-\frac{2 I_{m}}{T} \bmod \left(n^{T}+\frac{i-1}{3} p p\right) \frac{p}{2}<\bmod \left(n^{T}+\frac{i-1}{3}, p\right)<p
\end{array}\right.
$$

where, $I_{j}^{T}\left(n^{T}, m^{T}\right)$ are the gray-scales of projector pixel $\left(n^{T}, m^{T}\right)$ in three-step shifting patterns, respectively and $i=1$ 2.3. $\%$ is the coding-pitch of one triangular. $I_{\max }$ and $I_{\min }$ are the minimum and maxinum gray-scales of the triangular patterns. $\bmod \left(n^{T}, p\right)$ equals the remainder of $n^{T}$ eongruent modulo $p$.

The patterns $I_{i}^{p}$ projected from DVP is distorted by the projector gamma, and expressed as

where $f\left(\right.$ ) is the DVP response for input as $I_{i}^{T}$. The $I_{i}^{p}$ and ambient luminance $I^{A}$ is reflected by $3 \mathrm{D}$ object surface, and the reflected light $I_{i}^{O}$ modulated by $r$ the surface reflectance is expressed as

$$
I_{i}^{O}=r\left(I_{i}^{P}+I^{A}\right)
$$

and $I_{i}^{C}$ the gray-scale of distortion fringe image by CCD camera will be decoding in a computer, $1_{i}^{C}$ expresses as

$$
I_{i}^{C}=g\left(I_{i}^{o}\right)
$$

where $g()$ is the CCD camera response function including the nonlinear distortion. As defining above, the nonlinear relationship between the fringe image gray-scale with the triangular pattern gray-scale can simply expressed as follow

$$
\left\langle I_{i}^{C}\right\rangle=\left\langle I_{i}^{T}\right\rangle^{\gamma_{S}}
$$


where $\left\langle I_{i}^{C}\right\rangle$ is the normalized intensity value of the captured fringe image $I_{i}^{C}$, and $\left\langle I_{i}^{T}\right\rangle$ is the intensity normalized value governed by formula (1). $\gamma_{s}$ is the gamma value of the entire system including DVP and CCD camera.

It can be seen from formula (6) that applying an gamma pre-coding, $1 / \gamma_{p}<1$, during the generation of ideal image $I_{i}^{T}$ may help to attenuate the gamma effect and thus enhance accuracy, even when the number of images is minimum, i.e., three. In this case,

$$
\left\langle I_{i}^{C}\right\rangle=\left[\left\langle I_{i}^{T}\right\rangle^{\frac{1}{\gamma_{p}}}\right]^{\gamma_{S}}
$$

In reality, considering the stable ambient luminance, gray-scale amplitude, and system complexity, the desired gray-scale can be determined by the following formula

$$
\left\langle I_{i}^{C}\right\rangle=c_{1}\left\langle I_{i}^{T} \sum^{\frac{\gamma_{1}}{\gamma_{p}}+\gamma_{2}}+c_{y_{2}}\right\rangle
$$

where $\gamma_{1} / \gamma_{p}+\gamma_{2}=\gamma_{S}$ is the gamma value of the entire system including DVP and CCD camera. $\gamma_{1}$ and $\gamma_{2}$ are the parameters dependent the TPPSP system structure. And different $\gamma_{p}$ is corresponding different $\gamma_{s}$. The ideal of gamma pr-coding is detected the optimal $\gamma_{p}$ so that $\gamma_{s}=1 . c_{1}$ is the gray-scale modulation independent on any gamma value of the system, $c_{2}$ is the normalized gray-scale value of stableambienthuminance.

\subsection{Processing of gamma pre-coding}

The computer generates Suniform Muminance patterns, which all pixels have the same gray-scale. Then the uniform luminance patterns are projected by DVP onto a white plane. The CCD camera captures 3 image could respectively express as

The solution lof equations (9) is

$$
\left\{\begin{array}{l}
\left\langle I_{1}^{C}\right\rangle=c_{1}\left\langle I_{1}^{T}\right\rangle^{\gamma_{S}}+c_{2} \\
\left\langle I_{2}^{C}\right\rangle=c_{1}\left\langle I_{2}^{T}\right\rangle^{\gamma_{S}}+c_{2} \\
\left\langle I_{3}^{C}\right\rangle=c_{1}\left\langle I_{3}^{T}\right\rangle^{\gamma_{S}}+c_{2}
\end{array}\right.
$$

$$
\frac{\left\langle I_{1}^{C}\right\rangle-\left\langle I_{2}^{C}\right\rangle}{\left\langle I_{2}^{C}\right\rangle-\left\langle I_{3}^{C}\right\rangle}=\frac{\left\langle I_{1}^{T}\right\rangle^{\gamma_{S}}-\left\langle I_{2}^{T}\right\rangle^{\gamma_{S}}}{\left\langle I_{2}^{T}\right\rangle^{\gamma_{S}}-\left\langle I_{3}^{T}\right\rangle^{\gamma_{S}}}
$$

But the formula (10) is a transcendental equation, could not find the analytical solution. Defining estimate function as

$$
T\left(\gamma_{S}\right)=\frac{\left\langle I_{1}^{C}\right\rangle-\left\langle I_{2}^{C}\right\rangle}{\left\langle I_{2}^{C}\right\rangle-\left\langle I_{3}^{C}\right\rangle}-\frac{\left\langle I_{1}^{T}\right\rangle^{\gamma_{S}}-\left\langle I_{2}^{T}\right\rangle^{\gamma_{S}}}{\left\langle I_{2}^{T}\right\rangle^{\gamma_{S}}-\left\langle I_{3}^{T}\right\rangle^{\gamma_{S}}}=0
$$

There are two gamma pre-coding $\gamma_{p 1}$ and $\gamma_{p 2}$, totally six uniform luminance patterns, for finding $\gamma_{S 1}$ and $\gamma_{S 1}$ the numerical solution of equation (11). Then two group $\gamma_{p i}$ and $\gamma_{S i}$ can be expressed by homogeneous equation as 


$$
\left[\begin{array}{cc}
\frac{1}{\gamma_{p 1}} & 1 \\
\frac{1}{\gamma_{p 2}} & 1
\end{array}\right]\left[\begin{array}{l}
\gamma_{1} \\
\gamma_{2}
\end{array}\right]=\left[\begin{array}{l}
\gamma_{S 1} \\
\gamma_{S 2}
\end{array}\right]
$$

Once $\gamma_{1}$ and $\gamma_{2}$ are obtained, setting

$$
\gamma_{p}=\frac{1-\gamma_{2}}{\gamma_{1}}
$$

as an appropriate pre-coding value to the projected patterns. The same gamma pre-coding detection is carried on all pixels of triangular patterns. Apply gamma pre-coding with $\gamma_{p}$ during triangular pattern generation for all future measurements with the current setup.

\subsection{Precessing of ACDC obtained by linear least square}

In TPPSP, only three triangular patterns phase-s iifted by one third of the pitch are used to reconstruct the 3-D object. Definite $I_{j}^{c}\left(n^{c}, m^{c}\right)$ as the gray-scales of CCD camera image $\operatorname{pixel}\left(n^{c}, m^{c}\right)$. The matching pixel in projector image to $\left.f^{c}, m^{c}\right)$ could be found. And its row coordinate $n^{T}$ (namely absolute coordinate as follow) is wrapped into one coding-pitch as $n^{r} . n^{r}$ is wrapped coofdinate and calculates according formula (14). The camera image plane coordinates $\left(n, m^{c}\right)$ is omitted in formula (14).

$$
\begin{aligned}
& \frac{p}{6} \frac{\frac{p}{2} I_{1}^{C}-I_{2}^{C}-2 I_{3}^{C}}{I_{1}^{C}-I_{3}^{C}} \\
& \frac{p}{6} \frac{3 I_{1}^{C}-4 I_{2}^{C}+I_{3}^{C}}{I_{1}^{C}-I_{2}^{C}}
\end{aligned}
$$

Three difference coding-pitches are selected to obtain the absolute coordinate. If $0<p_{1}<p_{2}<p_{3}$ are three coding-pitches, and only if $n^{T}<\operatorname{lcm}\left\{p_{i}\right\}$ (where $\operatorname{lcm}\{\}$ denotes the Least Common Multiple), $n^{T}$ can be uniquely reconstructed from 3 wrapped coordinate $n_{i}^{r}$ as follow

$$
n^{T} \equiv\left(\sum_{i=1}^{3} n_{i}^{r} P_{i} p_{i}^{\prime}\right)(\bmod P)
$$


where $P=\operatorname{lcm}\left\{p_{i}\right\}, P_{i}$ defines as $P \times \operatorname{gcd}\left\{p_{1}, p_{2}, p_{3}\right\} / p_{i}$ (where $\operatorname{gcd}\{\}$ denotes the Greatest Common Divisor), $p_{i}^{\prime}$ is modular multiplicative inverse of $P_{i}$, i.e.

$$
P_{i} p_{i}^{\prime} \equiv 1\left(\bmod p_{i}\right)
$$

After gamma calibration using the gamma pre-coding, which is detail described in Section 2.1 and 2.2, the phase-shifting error from the gamma nonlinearity is suppressed to the minimum. But the light intensity is decayed with the optical path, and this decay will still induct the phase-shifting error. Fortunately, this error sours is linear, because CCD camera sample is the mean intensity of interest area of $3 \mathrm{D}$ object surface. The linear least square could be reduced this phase-shifting error.

Defining $n^{T_{m}}$ is the measurement value of $n^{T}$, and the linear relationship between $n^{T_{m}}$ and $n^{T}$ can be expressed as

$$
n^{T}=a n^{T_{m}}+b
$$

where $a$ and $b$ are the linear phase shifting error correction factors.

Assuming knowing $N$ point pairs $\left(n^{T_{m}}, n^{T}\right)$ the linear phase-shifting residuals error in $i$ th point pairs expressed as $\Delta_{i}=n_{i}^{T}-a n_{i}^{T_{m}}+b$. The sum of entire point pair residuals error is defined as $\Omega$

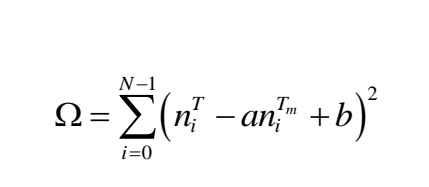

According to the principle of least squares, the linear phase shifting error correction factor are obtained as follow formula.

\section{3D Shape Measurement System and Experimental Results}

In order to verify the performance of the presented method in this paper, we programmed the 3D shape measurement experimental system of Figure 3. The system is composed of a Hitachi F22 camera with Computar M3Z1228C-MP camera lens and an Acer H7531D digital video projector. Camera images have a resolution of $1360 \times 1024$ pixels. The baseline between the camera and the projector is approximately $400 \mathrm{~mm}$. The angle between the optical axes of the camera and the projector is of about 30 degree.

Figure 4 shows the gray-scales response curve of this 3D shape measurement system. From this Figure, between 70 and 190, the gray-scale response is well, and the minimum and maximum gray-scales of the triangular patterns are selected respectively. The gamma pre-coding initial is $\gamma_{p 1}=2.5$ and $\gamma_{p 2}=3.5$. 3 normalized intensity values $I_{i}^{T}$, 
which is the uniform luminance patterns generated by computer 1 , are $I_{1}^{T}=0.4, I_{2}^{T}=0.5$ and $I_{3}^{T}=0.6$. The CCD camera images as shown in from Figure 5 to Figure 10.

The gamma pre-coding result is show in Figure 11. The brightness point is higher gamma value and the darkness is lower gamma value using in generation the 3D shape measurement triangular patterns by computer 1 . After gamma pre-coding calibration and ACDC linear compensation, the final absolute coordinate residuals error is shown as Figure 12. This data is come from absolute coordinate map of a plane.

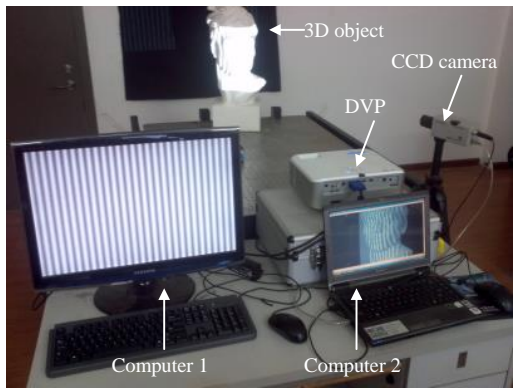

Figure 3. The setup of 3D shape measurement system

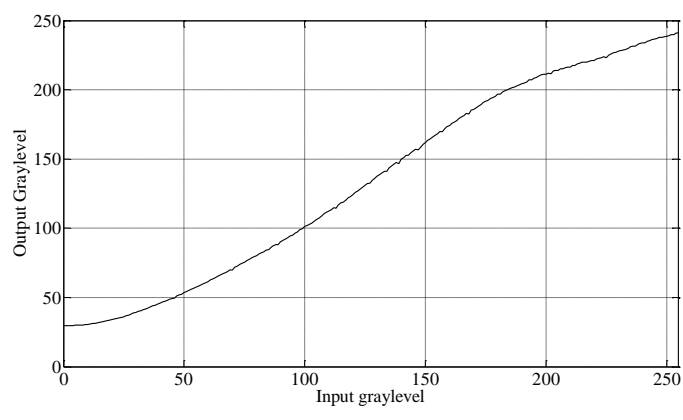

Figure 4. Measurement system Graylevei response function curve


Figure 5. Captured image by CCD camera $\gamma_{p 1}=2.5$, $I_{1}^{T}=0.4$

Figure 6. Captured image by CCD camera $\gamma_{p 1}=2.5, I_{2}^{T}=0.5$



Figure 9. Captured image by CCD camera $\gamma_{p 2}=3.5, I_{2}^{T}=0.5$

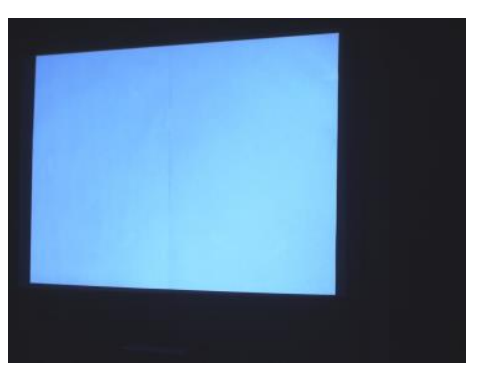

Figure 7. Captured image by CCD camera $\gamma_{p 1}=2.5, I_{3}^{T}=0.6$



Figure 10. Captured image by CCD camera $\gamma_{p 2}=3.5, I_{3}^{T}=0.6$ 




Figure 11. The pre-coding gamma for measuring triangular patterns

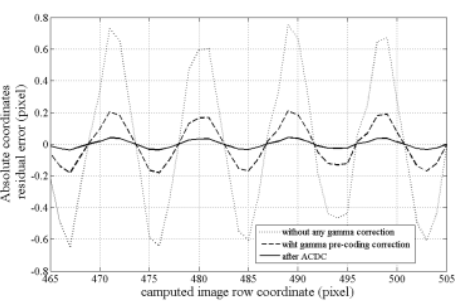

Figure 12. Final ACDC residuals

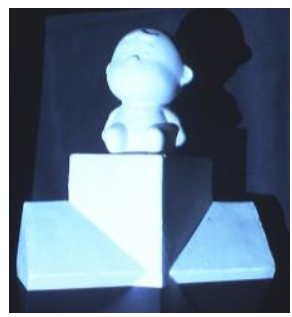

Figure 13. A tested plaster doll

In Figure 12, the dot-line is the absolute coordinate residuals error without any correction or compensation, the maximum residuals is 0.853 pixel. The dashed-line is the residuals error using pre-coding gamma values shown as Figure 1 , the maximum residuals is 0.237 pixel, the pre-coding gamma using 6 uniform/luminance patterns can reduce the absolute coordinate residuals error to $72.2 \%$. And the solid line is the residuals error using the method proposed in this paper, the maximum residuals is 0.052 , the total residuals reduction is $93.9 \%$.

In order to the method proposed, a complax curve surface, a tested plaster doll as shown in Figure 13, was reconstructed no using any correction or compensation shown as Figure 14 and Figure 15, respectively.From Figure 14, the reconstruction surface waviness is very clear, especially near the moath of doll. From Figure 15 this waviness from the nonlinearity or linearity arror source of the 3D shape measurement system is hardly any.

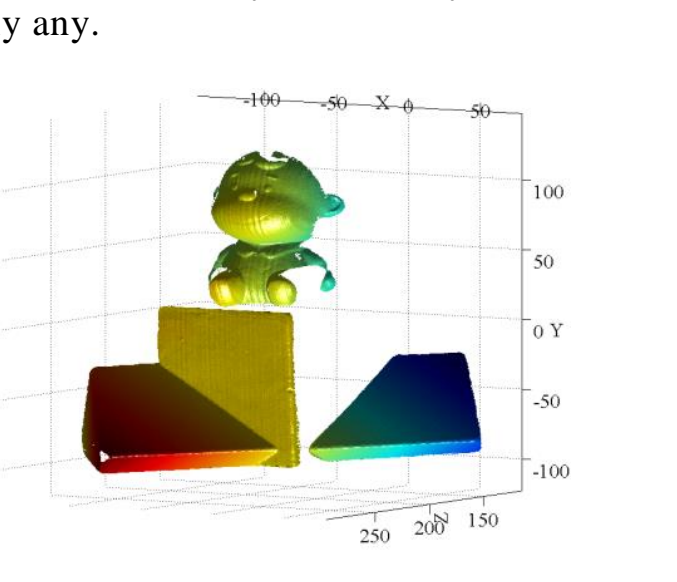

Figure 14. Reconstruction surface without any correction or compensation

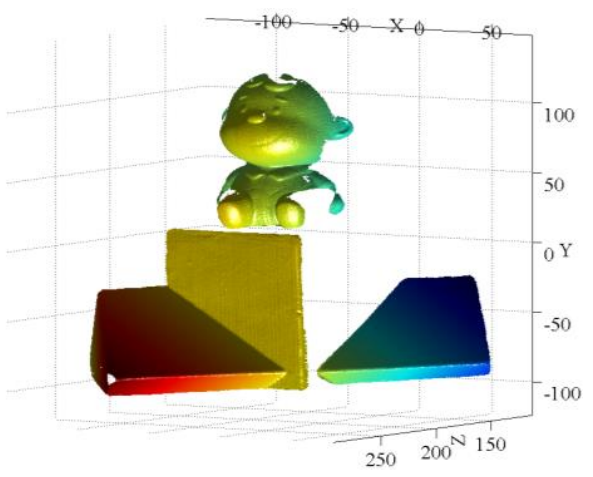

Figure 15. Reconstructed surface using the proposed method in this paper

\section{Conclusion}

A novel phase-shifting error reduction combined pre-coding gamma ideal with linear least square is presented in this paper. The proposed method achieving the gamma correction only using 6 uniform luminance patterns, the time-consuming in the same correction is fewer than other methods. The linear least square is further reducing the absolute coordinate distribution residuals error. The total reduction of absolute coordinate residuals is $93.9 \%$. The reconstruction surface of complex curve is hardly any the waviness, which is clearly display in the same reconstruction surface without any correction or compensation. 


\section{Acknowledgements}

This research is supported by Technology Research Project of Heilongjiang Province Education Department (12521080). The constructive comments from the reviewers are of this paper gratefully acknowledged which have helped the author to improve the paper.

\section{References}

[1] S. Zhang, Opt. Laser. Eng., vol. 2, no. 48, (2010).

[2] J. Salvi, S. Fernandez, T. Pribanic and X. Llado, Pattern Recogn., vol. 8, no. 43, (2010).

[3] Z. Li, Y. Shi, C. Wang, D. Qin and K. Huang, Opt. Commun., vol. 14, no. 282, (2009).

[4] L. C. Chen and X. L. Nguyen, Meas. Sci. Technol., vol. 5, no. 21, (2010).

[5] C. Waddington and J. Kofman, Opt. Laser. Eng., vol. 2, no. 48, (2010).

[6] P. Jia and J. Kofman and C. English, J. Opt. Soc. Am. A., vol. 10, no. 24, (2007).

[7] S. Zhang and P. S. Huang, Opt. Eng., vol. 6, no. 46, (2007).

[8] Y. Xu, L. Ekstrand, J. Dai and S. Zhang, Appl. Opt., vol. 17, no. 50, (2011).

[9] M. Carocci, S. Lazzari, R. Rodella and G. Sansoni, "3D range optical sensor: analysis of the measurement errors and development of procedures for their compensation", Proc.SPIE 3313. Three-Dimensional Image Capture and Applications, (1998) January 24; San Jose, CA.

[10] X. Chen, J. Xi and Y. Jin. Opt. Eng., vol. 11, no. 47, (2008)

[11] S. Zhang and S. Yau, Appl. Opt., vol. 1, no. 46, (2007).

[12] H. Guo, H. He and M. Chen, Appl. Opt., vol. 14, no 43 (2004). .

[13] T. Hoang, B. Pan, D. Nguyen and Z. Wang, Opt. Lett.) vol. 12, no/35, (2010).

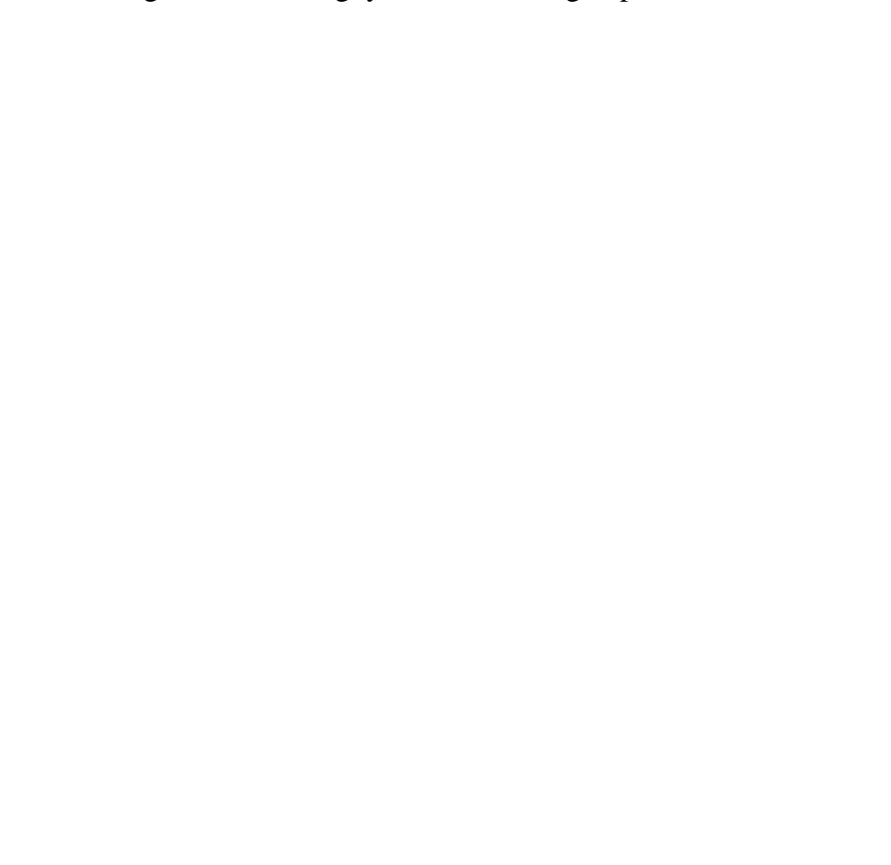


International Journal of Multimedia and Ubiquitous Engineering Vol.9, No.2 (2014)

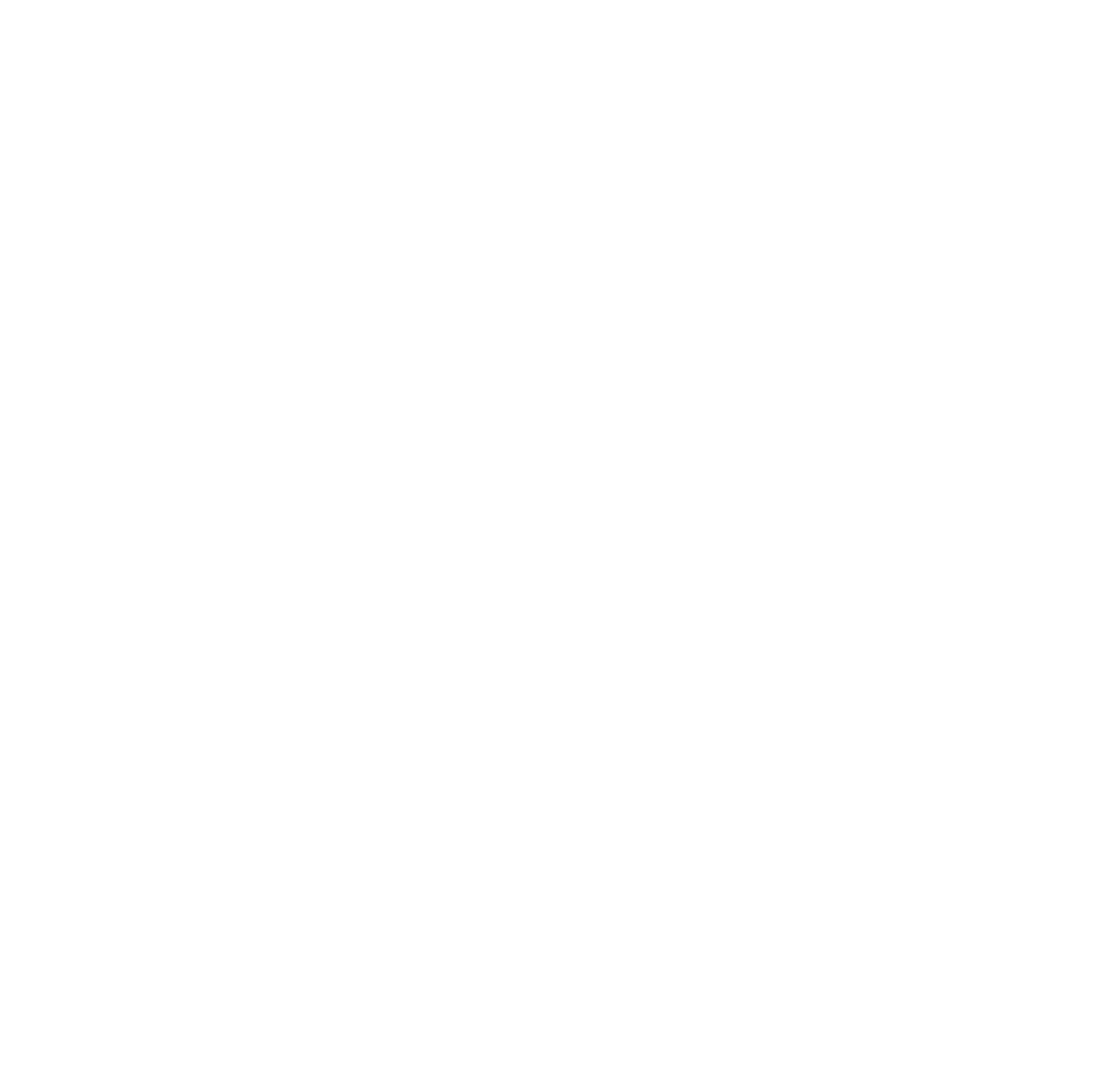

